\title{
THE PECULIAR PERIODIC YSO WL 4 IN $\rho$ OPHIUCHUS
}

\author{
Peter Plavchan, ${ }^{1,2}$ Alan H. Gee, ${ }^{1}$ Karl Stapelfeldt, ${ }^{2}$ and Andrew Becker ${ }^{3}$ \\ Received 2007 September 6; accepted 2008 July 25; published 2008 August 7
}

\begin{abstract}
We present the discovery of 130.87 day periodic near-infrared flux variability for the Class II T Tauri star WL 4 (=2MASS J16271848-2429059, ISO-Oph 128). Our data are from the 2MASS Calibration Point Source Working Database and constitute 1580 observations in $J, H$, and $K_{s}$ of a field in $\rho$ Ophiuchus used to calibrate the 2MASS All-Sky Survey. We identify a light curve for WL 4 with eclipse amplitudes of $\sim 0.4$ mag lasting more than one-quarter the period and color variations in $J-H$ and $H-K_{s}$ of $\sim 0.1 \mathrm{mag}$. The long period cannot be explained by stellar rotation. We propose that WL 4 is a triple YSO system, with an inner binary orbital period of 130.87 days. We posulate that we are observing each component of the inner binary alternately being eclipsed by a circumbinary disk with respect to our line of sight. This system will be useful in investigating terrestrial-zone YSO disk properties and dynamics at $\sim 1 \mathrm{Myr}$.
\end{abstract}

Subject headings: circumstellar matter — stars: pre-main-sequence — stars: variables: other

\section{INTRODUCTION}

When stars form and contract onto the main sequence, remnant material can remain in a circumstellar disk. More than half of the solar-type young stellar objects (YSOs) possess these primordial disks from which planets can eventually form (Meyer et al. 1997). The physical mechanisms responsible for the evolution and dissipation of primordial disks are not directly observed. $\rho$ Ophiuchus ( $\rho \mathrm{Oph}$ ) is a $\sim 135$ pe star-forming region containing several hundred $\sim 1$ Myr YSOs (Mamajek 2008; Natta et al. 2006; Barsony et al. 2005; Lada 1987; Lada \& Wilking 1984). Photometric variability is a common property of YSOs, and several large-sky and targeted variability studies of YSOs have been undertaken in the near-infrared (near-IR; Alves de Oliviera 2008; Barsony et al. 1997; Carpenter et al. 2001, 2002). With the Two Micron All-Sky Survey (2MASS) Calibration Point Source Working Database (Cal-PSWDB; Skrutskie et al. 2006), we are carrying out a program to study the near-IR variability of YSOs in $\rho$ Oph as a probe of stellar and circumstellar disk evolution. In this Letter, we present the discovery of a YSO with long-term periodic variability that we attribute to eclipses by a circumbinary disk.

WL 4 is a previously unremarkable $\sim 1$ Myr Class II T Tauri star in $\rho$ Oph (Natta et al. 2006; Strom et al. 1995). For WL 4, Natta et al. (2006) estimate a $J$-band extinction of $A_{J}=5.5$, an effective stellar temperature of $3715 \mathrm{~K}$, an intrinsic luminosity of $1.2 L_{\odot}$, and a mass of $0.45 M_{\odot}$. Natta et al. (2006) derive only upper limits for accretion from the $\mathrm{Pa} \beta$ and $\mathrm{Br} \gamma$ emission-line equivalent widths, implying WL 4 is not a strong accretor $\left(\log \left[\dot{M}_{\text {acc }} /\left(M_{\odot} \mathrm{yr}^{-1}\right)\right]<-9.1\right)$. For comparison, Strom et al. (1995) derived an extinction $A_{J}=5.0$ and $\sim 1$ Myr mass of $1.4 M_{\odot}$ from D'Antona \& Mazzitelli (1994) isochrones. Tsuboi et al. (2000) identify three consecutive X-ray flares with a quasi-period of $\sim 20$ hr. Ratzka et al. (2005) report a $0.176 "$ companion from high angular resolution imaging, with a projected separation of $\sim 24 \mathrm{AU}$ and a flux density ratio of $0.602 \pm 0.062$. Observations of WL 4 at infrared and sub-mm wavelengths are presented in $\S 2.2$.

\footnotetext{
${ }^{1}$ Infrared Processing and Analysis Center, California Institute of Technology, M/C 100-22, 770 South Wilson Avenue, Pasadena, CA 91125.

${ }^{2}$ Jet Propulsion Laboratory, California Institute of Technology, MS 183900, 4800 Oak Grove Drive, Pasadena, CA 91109.

${ }^{3}$ Astronomy Department, University of Washington, Seattle, WA 98195.
}

\section{OBSERVATIONS}

\subsection{MASS Calibration Observations}

2MASS imaged the entire sky in three near-IR bands between 1997 and 2001. Photometric calibration for 2MASS was accomplished using hourly observations of 35 selected calibration fields, with different fields visited each hour. One of these fields in $\rho$ Oph covers a region $8.5^{\prime}$ wide in R.A. by $60^{\prime}$ long in decl., centered at (R.A., decl. $)=\left(246.80780^{\circ},-24.68901^{\circ}\right)$. The calibration fields were observed using the same "freezeframe" scanning strategy used for the main survey that yielded a net $7.8 \mathrm{~s}$ exposure on the sky per scan, with six scans of the field taken in alternating declination directions during each hourly calibration observation. In three $\sim 6$ month visibility windows spanning 901 days, 1582 independent scans were made of the field in $\rho$ Oph, including 1580 detections of WL 4. The raw imaging data from each scan of a calibration field were reduced using the same pipeline used to process the main survey. All source extractions from all scans were loaded into the Cal-PSWDB (Cutri et al. 2006). We do not identify the cause of the two missing detections for WL 4 in the CalPSWDB, but it is likely an artifact of the automated processing.

\subsection{Thermal Infrared and Submillimeter Photometry}

The Cores to Disks (c2d) Spitzer Space Telescope Legacy program surveyed star-forming regions including $\rho$ Oph (Evans et al. 2003). The final c2d data delivery (DR4) includes measured flux densities from observations of WL 4 with MIPS with two epochs at $24 \mu \mathrm{m}$, one epoch each at 70 and $160 \mu \mathrm{m}$ (Padgett et al. 2008; Rieke et al. 2004), and two epochs with IRAC at 3.6, 4.5, 5.8, and $8.0 \mu \mathrm{m}$ (L. Allen et al. 2008, in preparation; Fazio et al. 2004). Padgett et al. (2008) notes that WL 4 and ISO-Oph 129 fall within an elliptical ring of $24 \mu \mathrm{m}$ emission $\sim 1^{\prime}$ in diameter centered on ISO-Oph 125 and 124. Barsony et al. (2005) observed WL 4 at 10.8 and $12.5 \mu \mathrm{m}$. The detection at $12.5 \mu \mathrm{m}$ appears to be inconsistent with the other measurements. Observations at $70 \mu \mathrm{m}$ with MIPS and in the sub-mm as reported in Andrews \& Williams (2007) and Stanke et al. (2006) are confusion limited by emission from ISO-Oph 124, 125, and 129. In our analysis, we treat such photometry as upper limits. 

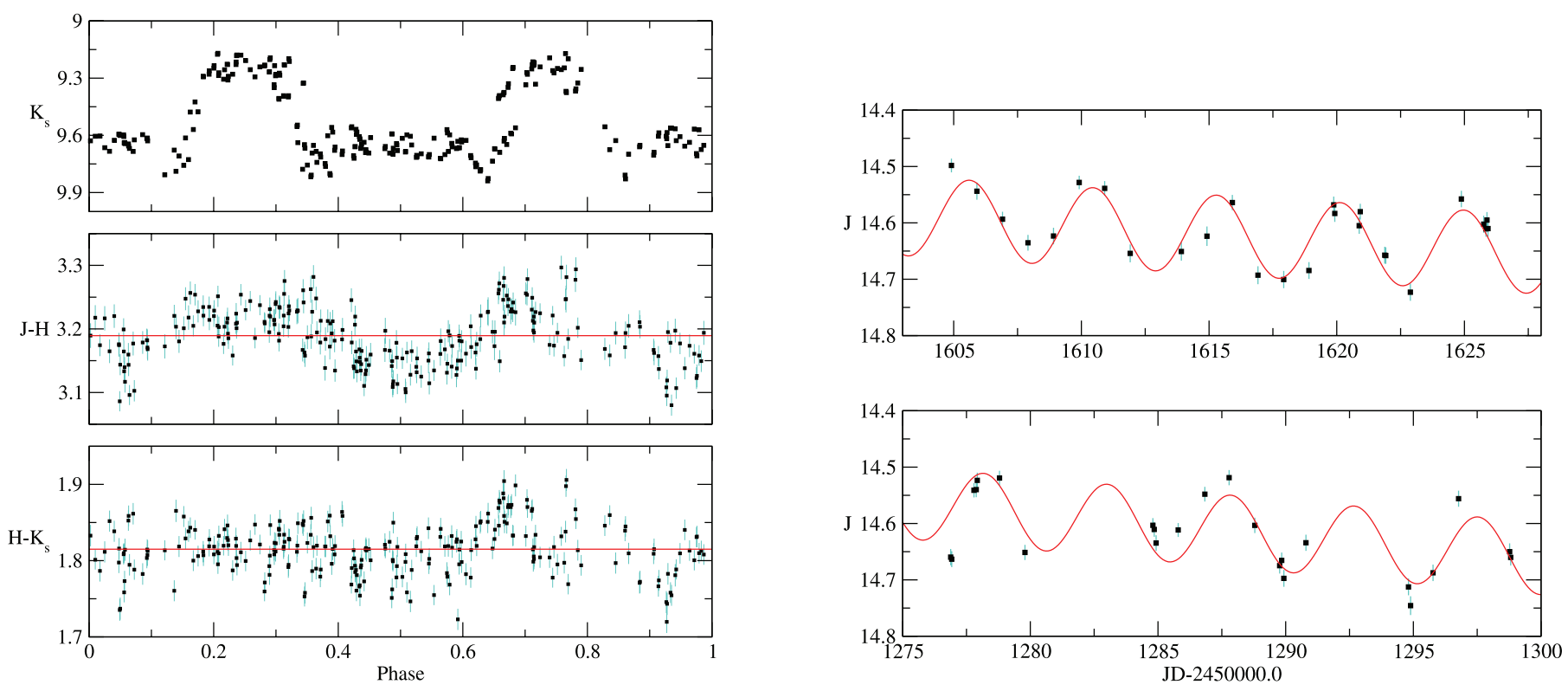

FIG. 1.-Left: (Top) $K_{s}$-band Cal-PSWDB light-curve data in black for WL 4. (Middle) $J-H$ Cal-PSWDB color curve. (Bottom) $H-K_{s}$ Cal-PSWDB color curve. Data are folded to a period of 130.87 days and plotted as a function of period phase. Right: Portions of the $J$-band light-curve data during the faint state to show the starspot variability modulated by the $\sim 5$ day stellar rotation period. Overlaid in red are the best-fitting sinusoids plus linear ramps, which reduce the photometric scatter of $\sim 0.07 \mathrm{mag}$ by a factor of $\sim 2$. The residuals are structured and indicate that the linear ramps are an oversimplification; we do not identify the physical origin of these variations. The sinusoids have different phases for all of the spans of faint or bright states that we investigate, implying starspot evolution with a coherence timescale longer than the rotation period and on the order of the binary period. Sinusoidal variations are largest in amplitude at $J$ band relative to $K_{s}$ band. For all panels, each group of six scans from a single hourly calibration observation are co-added, and $1 \sigma$ error bars are shown in teal.

\section{ANALYSIS AND RESULTS}

\subsection{Periodic Variability}

For WL 4, periodic variability is apparent in the unphased data, alternating between bright and faint states. We identify a period of $130.87 \pm 0.40$ days using both the Lomb-Scargle periodogram (Scargle 1982) and the period-searching algorithm of Plavchan et al. (2008). The phased light curve is shown in Figure 1, left. For JD $=2,450,000.0$, the corresponding phase in Figure 1 , left, is 0.33 . We find a peak-to-peak variation of $\sim 0.4 \mathrm{mag}$ in $J, H$ and $K_{s}$, with a corresponding reddening of $\sim 0.06$ mag in $J-H$ and $H-K_{s}$ when WL 4 is in the bright state. While a $\sim 65$ day period is consistent with the 2MASS results, our best physical model for the system $(\S 3.3)$ requires that this is an alias of the true period. The period is too long to be associated with a $\sim 1$ Myr YSO stellar rotation period (Rebull 2001).

Superposed on the long-term variability, we identify a second significant period of variability at $4.839 \pm 0.015$ days (Fig. 1 , right). We observe this additional periodic variability in both the faint and bright states, with a larger amplitude in $J$ relative to $K_{s}$. This variability is consistent with rotationally modulated stellar variability (Rebull 2001).

\subsection{Spectral Energy Distribution}

The c2d IRAC observations of WL 4 were fortunate to coincide with the bright and faint states predicted from the CalPSWDB data. We model the spectral energy distribution (SED) in both states. We fit by inspection the photometry with reddened PHOENIX NextGen (Hauschildt et al. 1999) synthetic stellar spectra and a two-temperature blackbody dust model. For both states of WL 4, we are able to reproduce the observations (Fig. 2). We are able to confirm the presence of an infrared excess associated with a primordial disk. We summarize the model parameters in Table 1 .

\subsection{Model}

The long period of the near-IR variability necessitates a binary companion, and the SED indicates the presence a primordial disk. We denote the binary components WL 4a and WL $4 \mathrm{~b}$ and the companion resolved in Ratzka et al. (2005) as WL 4c. All three components are unresolved with 2MASS and Spitzer. The observed IRAC variability implies a circumbinary disk around WL $4 \mathrm{ab}$, but part of the infrared excess could be associated with a disk around WL 4c. To explain the shape of the light curve, we postulate that a component of the WL $4 \mathrm{ab}$ binary goes into obscuration and reemerges from behind a circumbinary disk every 65.44 days. The binary must be inclined with respect to the disk and the disk relatively close to edge-on with respect to our line of sight.

We can solve for the brightnesses of the three components using the total stellar luminosity derived from the SED fit, the magnitude depth of the faint state from one component being obscured, and the flux ratio observed during a predicted bright state in Ratzka et al. (2005). We find that WL 4 is composed of three approximately equal brightness $0.6 L_{\odot}$ YSOs. The symmetry between the brightnesses of WL $4 \mathrm{a}$ and WL $4 \mathrm{~b}$ implies that the binary period is 130.87 and not 65.44 days. Our model predicts that WL $4 \mathrm{a}$ and WL $4 \mathrm{~b}$ alternate being obscured by the circumbinary disk. The similar depth and system color during consecutive faint states is consistent with similar spectral types for WL $4 \mathrm{a}$ and WL $4 \mathrm{~b}$. The color variations occur during the ingress and egress phases of the eclipses, and we do not model this additional complexity. The color changes may indicate a slight difference in spectral types for WL $4 \mathrm{a}$ and WL $4 \mathrm{~b}$ or may be due to varying scattered light 


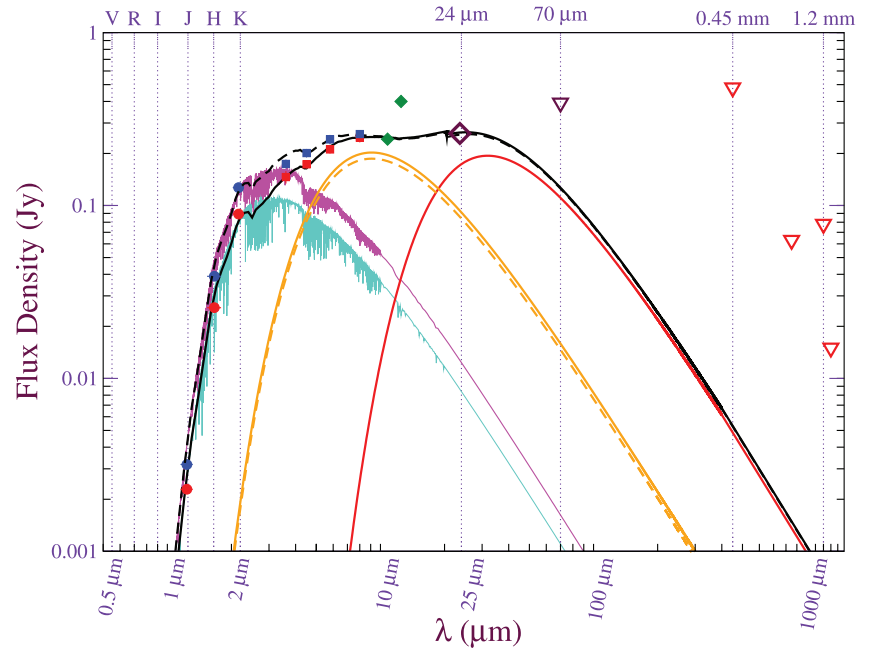

FIG. 2.-Spectral energy distribution for WL 4. Data: Red circles: 2MASS Cal-PSWDB flux densities in the faint state (Fig. 1, left, phase 0); blue circles: 2MASS Cal-PSWDB flux densities in the bright state (phase 0.25); red squares: c2d IRAC flux densities in the faint state (JD = 2,453,071.831, Fig. 1, left, phase 0.142); blue squares: c2d IRAC flux densities in the bright state (JD $=2,453,092.736,0.302)$; green diamonds: 10.8 and $12.5 \mu \mathrm{m}$ measurements (JD $=\sim 2,450,198.0,0.183$; JD $=\sim 2,451,358.0,0.047$, Barsony et al. 2005); purple diamond: c2d MIPS $24 \mu \mathrm{m}$ flux density at both epochs (JD = $2,453,083.888,0.235 ; \mathrm{JD}=2,453,084.113,0.236$ ); purple triangle: $\mathrm{c} 2 \mathrm{~d}$ MIPS $70 \mu \mathrm{m}$ flux density upper limit; red triangles: sub-mm flux density upper limits (Andrews \& Williams 2007; Stanke et al. 2006). The IRAC epochs correspond to the channel 1 and 3 observations. The channel 2 and 4 observations were taken within $1 \mathrm{hr}$ of these times. Model: Solid red line: cold-dust component; solid orange line: hot-dust component in the faint state; dashed orange line: hot-dust component in the bright state; cyan line: reddened synthetic spectra for the two WL 4 components unobscured in the faint state; magenta line: reddened synthetic spectra for the three WL 4 components unobscured in the bright state; black solid line: sum of stellar components and hot-dust and colddust spectra in the faint state; black dashed line: sum of stellar components and hot-dust and cold-dust spectra in the bright state.

flux. The estimated stellar masses imply a binary separation of $0.47 \mathrm{AU}$, or $\sim 50$ stellar radii. Finally, we attribute the 4.84 day periodic variability to stellar rotation modulated starspots on WL $4 \mathrm{c}$, because this is consistent with the variability being observed in both states.

\section{DISCUSSION}

We list supporting evidence for our model in $\S \S 4.1-4.3$.

\subsection{Derived Model Parameters and Lack of Detected Accretion Signatures}

The dust luminosities we derive are sufficient to justify the shadowing model, and the variability is detected from 1 to $8 \mu \mathrm{m}$. Only two parameters of our model are necessarily varied between the bright and faint states to reproduce the SED: the hot-dust luminosity which is important to reproduce the IRAC channels 2-4 photometry, and the obscuration of one stellar component in the faint state. The change in the hot-dust luminosity indicates possible dynamical interaction or disk "warping," or simply changing illumination/heating. The hotdust temperature is consistent with the stable inner orbital radius of $\sim 2-3$ times the binary semimajor axis (Harrington 1977). The presence of WL $4 c$ could account for the dynamical origin of a disk inclined with respect to the orbit of the inner WL 4ab binary.
TABLE 1

SED Model Parameters

\begin{tabular}{|c|c|}
\hline Parameter & Value \\
\hline \multicolumn{2}{|l|}{ Fixed } \\
\hline $\begin{array}{l}\text { Distance } \ldots \ldots \ldots \ldots \ldots \ldots \ldots \ldots \ldots \\
\text { Short } \lambda \text { extinction power law } \ldots \ldots \ldots \\
\text { Long } \lambda \text { extinction power law } \ldots \ldots \ldots \\
\text { Extinction power-law transition } \ldots \ldots \\
T_{b}, T_{c}, R_{b}, R_{c}, M_{b}, M_{c} \ldots \ldots \ldots \ldots \ldots \ldots\end{array}$ & $\begin{array}{l}135 \mathrm{pc} \\
-1.7^{\mathrm{a}} \\
-1^{\mathrm{a}} \\
3.5 \mu \mathrm{m}^{\mathrm{a}} \\
T_{a}, R_{a}, M_{a}^{\mathrm{b}}\end{array}$ \\
\hline \multicolumn{2}{|c|}{ Varying, Best Fit } \\
\hline $\begin{array}{l}\text { WL } 4 A_{J} \text { extinction } \ldots \ldots \ldots \ldots \ldots \ldots \ldots \\
T_{a}, R_{a}, M_{a} \ldots \ldots \ldots \ldots \ldots \ldots \ldots \ldots \ldots \ldots \\
\text { Cold-dust } T, L \ldots \ldots \ldots \ldots \ldots \ldots \ldots \ldots \\
\text { Hot-dust } T, L \text { bright state } \ldots \ldots \ldots \ldots \ldots \\
\text { Hot-dust } T, L \text { faint state } \ldots \ldots \ldots \ldots \ldots \ldots\end{array}$ & $\begin{array}{l}6 \mathrm{mag} \\
3600 \mathrm{~K},{ }^{\mathrm{c}} 2.0 R_{\odot}, 0.40 M_{\odot}^{\mathrm{d}} \\
160 \mathrm{~K},{ }^{\mathrm{e}} 0.017 L_{\odot} \\
560 \mathrm{~K},{ }^{\mathrm{e}} 0.055 L_{\odot} \\
560 \mathrm{~K},{ }^{\mathrm{e}} 0.061 L_{\odot} \\
\end{array}$ \\
\hline $\begin{array}{l}{ }^{\mathrm{a}} \text { Extinction wavelength dependence } \\
\text { Becklin et al. } 1978 . \\
{ }^{\mathrm{b}} \text { An equal-mass triple-system model } \\
{ }^{\mathrm{c}} \text { Fit to nearest } 100 \mathrm{~K} \text {. } \\
{ }^{\mathrm{d}} \text { Estimated from } 1 \mathrm{Myr} 3600 \mathrm{~K} \text { Sies } \\
{ }^{\mathrm{e}} \text { Fit to nearest } 5 \mathrm{~K} \text {. }\end{array}$ & $\begin{array}{l}\text { adopted from Mathis 1990; } \\
\text { is used ( } \$ 3.3 \text { ). } \\
\text { isochrone (Siess et al. 2000). }\end{array}$ \\
\hline
\end{tabular}

The lack of a significant component of hot $\sim 1000 \mathrm{~K}$ dust and the lack of strong accretion signatures in Natta et al. (2006) imply the lack of a massive circumprimary disk around WL $4 \mathrm{a}$ or $4 \mathrm{~b}$. The lack of detected accretion also implies that periodically driven accretion by the companion is not a favored scenario, such as is hypothesized for DQ Tau and AA Tau (Mathieu et al. 1997; Bouvier et al. 2003). Finally, the apparent $K_{s}$ magnitude of WL 4 is consistent with a directly visible $\rho$ Oph YSO and is too bright to be an edge-on disk system seen only in scattered light (K. Stapelfeldt et al. 2008, in preparation; Watson \& Stapelfeldt 2007; Stapelfeldt et al. 1997).

\subsection{Duration of Faint State}

A stable circumstellar disk around WL $4 \mathrm{a}$ or $4 \mathrm{~b}$ with an outer radius of one-third the binary separation (Artymowicz \& Lubow 1994) would produce an eclipse only $10 \%$ of the period, so this configuration can be ruled out. Excepting for the $\sim 4.84$ day periodic variability, the light curves in the faint and bright states are relatively flat and smoothly varying for $\sim 25$ and $\sim 13$ days, respectively. The transition between bright and faint states, including the "kinks" in the light curve at phases of $0.14,0.36,0.63$, and 0.86 , last $\sim 13$ days apiece. We speculate that the "kinks" could be due to disk substructure such as a puffed-up or overdense inner edge.

\subsection{Similarities to $\mathrm{KH}-15 \mathrm{D}$}

$\mathrm{KH}-15 \mathrm{D}$ is a unique K6-K7 binary YSO in NGC 2264 (Kearns \& Herbst 1998; Badalian \& Erastova 1970). Every $\sim 48$ days, KH-15D periodically varies in brightness by $\sim 4 \mathrm{mag}$ between relatively quiescent bright and faint states. An inclined binary with a $\sim 48$ day period surrounded by a nearly edge-on circumbinary disk is invoked to explain these observations, as the primary component of $\mathrm{KH}-15 \mathrm{D}$ periodically "peeks out" from behind the disk (Winn et al. 2006; Johnson et al. 2005; Winn et al. 2004; Chiang \& Murray-Clay 2004; Johnson \& Winn 2004; Winn et al. 2003). The near-IR photometry and colors vary in tandem in a similar fashion for both KH-15D and WL 4, although the amplitude differs. When both sources get brighter, both sources also get redder in $J-H$ and $H-K_{s}$ (Kusakabe et al. 2005). Kusakabe et al. (2005) attribute the 
reddening for $\mathrm{KH}-15 \mathrm{D}$ to changes in the scattered light flux. Similar features such as the "kinks" in the light curve in Figure 1 , left, have also evolved with time for $\mathrm{KH}-15 \mathrm{D}$.

\section{CONCLUSIONS AND FUTURE WORK}

We have identified periodic variability for the YSO WL 4 in $\rho$ Oph that is likely due to alternating eclipses of two different components of a binary system by a circumbinary disk. The faint state lasts longer than the bright state, ruling out a coplanar stable circumprimary disk to explain the observations. WL 4 is a unique and valuable probe of YSO terrestrial-zone disk evolution. We want to confirm our binary model for the lightcurve modulation. The radial velocity amplitude implied by our model should be measurable through high-resolution nearIR spectroscopy with adaptive optics. Detailed modeling of the disk with orbital dynamics of the binary is warranted to reproduce the observed light curves, including the "kinks," and to investigate the dynamical stability of our model. Near- and mid-IR photometric and spectroscopic observations will enable a characterization of the dust grain properties and disk structure.

This publication makes extensive use of data products from 2MASS, which is a joint project of the University of Massachusetts and IPAC/California Institute of Technology, funded by NASA and NSF. This research has made use of the NASA/ IPAC Infrared Science Archive, which is operated by Jet Propulsion Laboratory, California Institute of Technology, under contract with NASA. Thanks to Mike Meyer, Mike Werner, Angelle Tanner, and Eric Agol for their conversations and comments. Parts of the research described in this publication were carried out at JPL.

\section{REFERENCES}

Alves de Oliviera, C., \& Casali, M. 2008, A\&A, 1, 155

Andrews, S., \& Williams, J. 2007, ApJ, 671, 1800

Artymowicz, P., \& Lubow, S. 1994, ApJ, 421, 651

Badalian, H., \& Erastova, L. 1970, Astron. Tsirk., 591, 4

Barsony, M., Kenyon, S., Lada, E., \& Teuben, P. 1997, ApJS, 112, 109

Barsony, M., Ressler, M., \& Marsh, K. 2005, ApJ, 630, 381

Becklin, E., Matthews, K., Neugebauer, G., \& Willner, S. 1978, ApJ, 220, 831

Bouvier, J., et al. 2003, A\&A, 409, 169

Carpenter, J., Hillenbrand, L., \& Skrutskie, M. 2001, AJ, 121, 3160

Carpenter, J., Hillenbrand, L., Skrutskie, M., \& Meyer, M. 2002, AJ, 124, 1001

Chiang, E. I., \& Murray-Clay, R. A. 2004, ApJ, 607, 913

Cutri, R., et al. 2006, Explanatory Supplement to the 2MASS All Sky Data Release and Extended Mission Products (Pasadena: IPAC), http://www.ipac .caltech.edu/2mass/releases/allsky/doc/

D’Antona, F., \& Mazzitelli, I. 1994, ApJS, 90, 467

Evans, N. J., et al. 2003, PASP, 115, 965

Fazio, G. G., et al. 2004, ApJS, 154, 39

Harrington, R. S. 1977, AJ, 82, 753

Hauschildt, P., Allard, F., \& Baron, E. 1999, ApJ, 512, 377

Johnson, J. A., \& Winn, J. N. 2004, AJ, 127, 2344

Johnson, J. A., et al. 2005, AJ, 129, 1978

Kearns, K., \& Herbst, W. 1998, AJ, 116, 261

Kusakabe, N., et al. 2005, ApJ, 632, L139

Lada, C. J. 1987, in IAU Symp. 115, Star Forming Regions, ed. M. Peimbert \& J. Jugaku (Dordrecht: Reidel), 1
Lada, C. J., \& Wilking, B. A. 1984, ApJ, 287, 610

Mamajek, E. 2008, Astron. Nachr., 329, 10

Mathieu, R. D., et al. 1997, AJ, 113, 1841

Mathis, J. 1990, ARA\&A, 28, 37

Meyer, M. R., et al. 1997, AJ, 114, 288

Natta, A., Testi, L., \& Randich, S. 2006, A\&A, 452, 245

Padgett, D., et al. 2008, ApJ, 672, 1013

Plavchan, P., et al. 2008, ApJS, 175, 191

Ratzka, T., Köhler, R., \& Leinert, Ch. 2005, A\&A, 437, 611

Rebull, L. 2001, AJ, 121, 1676

Rieke, G., et al. 2004, ApJS, 154, 25

Scargle, J. 1982, ApJ, 263, 835

Siess, L., Dufour, E., \& Forestini, M. 2000, A\&A, 358, 593

Skrutskie, M., et al. 2006, AJ, 131, 1163

Stanke, T., et al. 2006, A\&A, 447, 609

Stapelfeldt, K., et al. 1997, in Proc. IAU Symp. 182, Herbi-Haro Flows and the Birth of Stars, ed. B. Reipurth \& C. Bertout (Dordrecht: Kluwer), 355

Strom, K., Kepner, J., \& Strom, S. 1995, ApJ, 438, 813

Tsuboi, Y., et al. 2000, Adv. Space Res., 25, 535

Watson, A., \& Stapelfeldt, K. 2007, AJ, 133, 845

Winn, J. N., Garnavich, P. M., Stanek, K. Z., \& Sasselov, D. D. 2003, ApJ, 593, L121

Winn, J. N., Holman, M. J., Johnson, J. A., Stanek, K. Z., \& Garnavich, P. M. 2004, ApJ, 603, L45

Winn, J. N., et al. 2006, ApJ, 644, 510 